\title{
Análisis Termogravimétrico y Estudio Cinético de la Pirólisis de Residuos Sólidos Veterinarios
}

\section{Thermogravimetric Analysis and Kinetic Study on Pyrolysis of Veterinarian Solid Waste}

\section{Andrés Felipe Rojas González ${ }^{1}$, Laura María Aranzazu Ríos ${ }^{2}$}

${ }^{1}$ Grupo de Investigación Aprovechamiento de Residuos, Departamento de Ingeniería Química, Universidad Nacional de Colombia, sede Manizales, Manizales, Colombia. correspondence email: anfrojasgo@unal.edu.co,

2 Jefe de Control de Calidad en la Planta de Alcoholes Sulfatados en la empresa Químicos del Cauca, Cauca, Colombia.

\section{Resumen}

Contexto: Los residuos institucionales de centros hospitalarios se pueden clasificar en residuos procedentes de instituciones de salud para la atención humana y para la atención de animales, estas últimas comúnmente llamadas veterinarias. En general estos residuos son peligrosos, por lo cual su disposición final es a través de la incineración. Dado que la mayoría son residuos orgánicos se puede aprovechar su poder energético en procesos de combustión o pirólisis. Este trabajo se motivó debido a que en la literatura no se encuentran artículos de cinética de pirólisis de residuos de veterinarias, enfocándose solo en residuos de hospitales de atención humana.

Método: Se caracterizó y estudió la cinética de pirólisis, por análisis termogravimétrico, de seis principales residuos veterinarios (gasas, hisopos, algodón, jeringas plásticas, pelo, uñas). La caracterización se realiza por análisis inmediato, elemental y análisis termogravimétrico. Se establecen las características de reactividad y de capacidad de pirólisis. El estudio de la cinética de pirólisis se llevó a cabo mediante la determinación del triplete cinético por el método isoconversional de Starink.

Resultados: Se estableció que el índice de pirólisis aumenta con la velocidad de calentamiento y que la degradación térmica depende del tipo de material del residuo. De igual manera se encontró que la temperatura $\left(\Delta T=T_{f}-T_{i}\right)$ para la descomposición térmica de los residuos veterinarios es: $\Delta T_{u \tilde{n} a s}>$ $\Delta T_{\text {pelo }}>\Delta T_{\text {hisopo }}>\Delta T_{\text {gasa }}>\Delta T_{\text {algodón }}>\Delta T_{\text {jeringa }}$, la energía de activación es $E_{\text {uñas }}>$ $E_{\text {pelo }}>E_{\text {jeringa }}>E_{\text {hisopo }}>E_{\text {gasa }}>E_{\text {algodón }} \mathrm{y}$ el orden de reacción es: $n_{\text {pelo }}>n_{\text {uñas }}>$ $n_{\text {hisopo }}>n_{\text {algodón }}>n_{\text {gasa }}>n_{\text {jeringa }}$.

Conclusiones: Estos resultados sugieren la posibilidad de aprovechar los residuos veterinarios para la generación de energía, proporcionando una alternativa de desarrollo energético sos- tenible a ciudades en continuo crecimiento, desde el punto de vista energético y ambiental.

Palabras clave: Índice de pirólisis, método de Starink, residuos veterinarios, termogravimetría, triplete cinético.

Idioma: Español

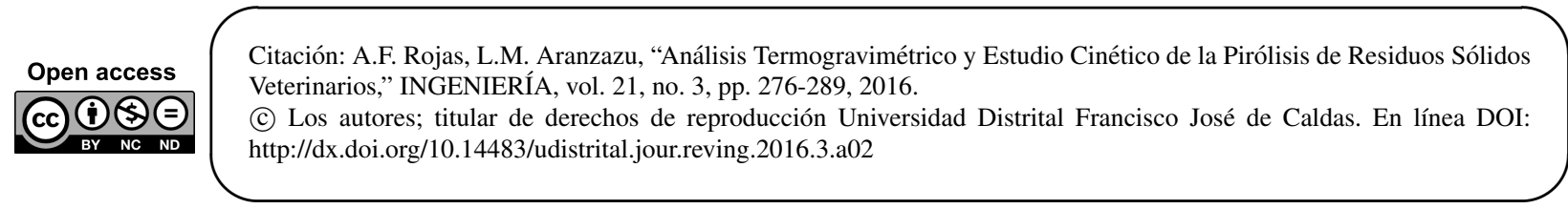




\begin{abstract}
Context: Institutional waste from clinical centers can be classified as those coming from health institutions dedicated to human attention and those coming from centers for animal veterinary care. The latter are mainly hazardous wastes, hence their disposal requires incineration. Most of such waste is organic, and it is possible, therefore, to take advantage of their energetic power in combustion or pyrolysis processes. This work is motivated because no literature was found on the pyrolysis kinetics veterinary waste, as this kind of studies are mainly focused on hospital waste of human health care .
\end{abstract}

Method: The kinetics of pyrolysis is characterized and studied by means of thermogravimetric analysis of 6 major veterinary waste (gauze, cotton swabs, cotton, nails, hair, plastic syringes). The characterization is performed by proximate and elemental analysis, and thermogravimetric analysis. Reactivity characteristics and pyrolytic capability of wastes are established. The kinetics study on pyrolysis was carried out by determining the kinetic triplet by isoconversional Starink method.

Results: It was established that the pyrolysis index increases with the heating rate and that the thermal degradation depends on the material type of the waste. Similarly, it was found that the temperature $\left(\Delta T=T_{f}-T_{i}\right)$ for the thermal decomposition of veterinary waste is: $\Delta T_{\text {nails }}>\Delta T_{\text {hair }}>$ $\Delta T_{\text {cotton swabs }}>\Delta T_{\text {gauze }}>\Delta T_{\text {cotton }}>\Delta T_{\text {plastic syringes }}$; the activation energy is $E_{\text {nails }}>E_{\text {hair }}>$ $E_{\text {plastic syringes }}>E_{\text {cotton swabs }}>E_{\text {gauze }}>E_{\text {cotton }}$, and the reaction order is: $n_{\text {hair }}>n_{\text {nails }}>$ $n_{\text {cotton swabs }}>n_{\text {cotton }}>n_{\text {gauze }}>n_{\text {plastic syringes. }}$.

Conclusions: These results suggest the possibility of using veterinary wastes for power generation, providing an alternative for sustainable energy development to cities in continuous growth, from both, energetic and environmental points of view.

Keywords: kinetic triplet, pyrolysis index, Starink method, thermogravimetry, veterinary wastes.

\title{
1. Introducción
}

La problemática en el manejo (recolección, transporte, almacenamiento y disposición final) de los desechos hospitalarios que enfrenta la humanidad, proviene del crecimiento exponencial de la población y el afán en alcanzar la industrialización de los países. El inadecuado manejo de los residuos puede generar daños físicos e infecciones graves en las personas que trabajan en hospitales, en pacientes, en recicladores y en la comunidad en general, al igual que en los animales, que de alguna manera tiene contacto con estos residuos [1]. Entre los residuos hospitalarios se encuentran: i) los residuos provenientes de instituciones hospitalarias (hospitales, clínicas médicas, centros de salud, ancianatos y clínicas de reposo) que atienden al ser humano, y ii) los residuos procedentes de centros veterinarios, que atienden toda clase de animales [2]. Los residuos hospitalarios o residuos médicos se definen como cualquier residuo sólido generado en el diagnóstico, tratamiento o inmunización de seres humanos o animales, al igual que los que resultan de investigaciones, en la producción o ensayos de productos biológicos [2].

Los residuos veterinarios se generan por el mantenimiento estético y médico de animales. Estos residuos dependen del tipo de veterinaria, ya que existen veterinarias rurales donde se atienden animales como perros, gatos, conejos, vacas, caballos, cerdos, gallinas, patos, burros, ovejas, cabras, entre otros, y las veterinarias urbanas dedicadas a la atención de mascotas como perros, gatos, pája- 
ros y hámsteres, entre los más comunes. En los objetos comúnmente encontrados en los residuos veterinarios se encuentran: tubos para transfusión, colectores de muestra para orina, guantes médicos, tapabocas, catéteres, algodones, gasas, toallas de papel, orina, piel, músculos, sabanas, ropa, medicamentos, envases plásticos y de vidrio y cajas de medicamentos [3] [4].

Tales residuos se han caracterizado por análisis termogravimétrico, para estudiar su comportamiento térmico en atmósfera oxidante (combustión) [5] [6] [7] y en atmósfera inerte (pirólisis) [4] [8]. De igual manera se ha evaluado la capacidad de reducción de volumen y de peligrosidad de los residuos, y la recuperabilidad de la energía contenida en ellos [9]. El comportamiento térmico en combustión y pirólisis, determinado por medio de un estudio cinético, generalmente se lleva a cabo por análisis termogravimétrico en atmósfera de nitrógeno (pirólisis) [10] y en atmósfera de aire (combustión) [7], bajo condiciones no isotérmicas. Esos estudios cinéticos se centran en la determinación del triplete cinético, el cual está constituido por los parámetros de energía de activación, orden de reacción y factor pre-exponencial/factor de frecuencia.

De todo lo anterior y como se sugiere en la literatura, es importante realizar investigaciones que lleven a los ingenieros a formular modelos energéticos diversificados, basados en el desarrollo de técnicas y tecnología más eficientes, lo cual se logra con el conocimiento en el comportamiento energéticos de los materiales [11], y buscando fuentes alternas de energía, que sean limpias y renovables, como los residuos de las clínicas veterinarias.

El objetivo de este trabajo es caracterizar y estudiar la cinética de pirólisis, por análisis termogravimétrico, de seis residuos (algodón, gasas, cuerpo de las jeringas, hisopos, pelo y uñas) de las clínicas y centros veterinarios urbanos de la ciudad de Manizales. La caracterización se realiza por medio del análisis inmediato, elemental y análisis termogravimétrico, el cual se realiza en atmósfera inerte y a cuatro velocidades de calentamiento $\left(5,10,20 \mathrm{y} 40^{\circ} \mathrm{C} / \mathrm{min}\right)$. A partir de los perfiles de pirólisis se obtiene información de las temperaturas de inicio, máxima y final de degradación, la velocidad máxima de pérdida de peso y delta de temperatura de degradación, y se establecen las características de reactividad por medio de las temperaturas características y de capacidad de pirólisis. El estudio de la cinética de pirólisis se llevó a cabo mediante la determinación del triplete cinético por medio del método isoconversional de Starink [10] [12].

\section{Materiales y métodos}

\subsection{Materiales}

Los residuos sólidos veterinarios se adquirieron de tres centros veterinarios, de veinticuatro existentes en la ciudad de Manizales. Estos residuos se recogieron en el mismo periodo de tiempo en las tres veterinarias, con un promedio en peso de $20 \mathrm{~kg}$ en cada una de ellas. Las tres veterinarias se escogieron por su importancia y cobertura de la ciudad. Una vez obtenidos los residuos de dichos centros, se identificaron los seis componentes mayoritarios, los cuales fueron: algodón, gasa, hisopo, jeringas, pelo y uñas. Adicionalmente a estas componentes por separado, también se analizó una muestra conocida como mezcla, la cual consiste en una muestra representativa de los residuos obtenidos en los tres centros veterinarios. Posterior a la identificación de los componentes 
individuales de los residuos veterinarios, se procedió a separarlos por tipos, pesarlos y posteriormente se redujeron de tamaño por corte y trituración, hasta un tamaño de partícula entre 0.1 y 1 mm. Para los respectivos análisis de cada uno de los residuos, se tomó una muestra triturada de $6 \mathrm{~g}$.

\subsection{Caracterización fisicoquímica de los residuos}

Los seis residuos se caracterización de acuerdo a las normas ASTM para análisis inmediato y elemental. En el análisis inmediato se determina el contenido de humedad total (ASTM D 3302), cenizas (ASTM D 7582), materia volátil (ASTM D 7582) y carbono fijo (ASTM D 3172). Este análisis se llevó a cabo en un analizador termogravimétrico marca LECO TGA-601. El poder calorífico superior (PCS) e inferior (PCI) se establece de acuerdo a la norma ASTM D 5865, empleando una bomba calorimétrica marca LECO AC 350. En el análisis elemental se cuantifica el contenido de carbono (ASTM D 5373), hidrógeno (ASTM D 5373), nitrógeno (ASTM D 5373), azufre (ASTM D 4239) y oxígeno (ASTM D 5373) de los residuos. La determinación de los elementos C, H y N se realizó en un analizador LECO CHN-268, el azufre se obtuvo en un sulfurómetro marca LECO S-632 y el oxígeno se calculó por diferencia incluido el contenido de cenizas.

\subsection{Caracterización termogravimétrica de los residuos}

La caracterización termogravimétrica se lleva a cabo en un analizador termogravimétrico Q600 simultáneo DSC-TGA TA Instrument. Con este análisis se obtuvieron los perfiles de pirólisis de cada residuo a cuatro velocidades de calentamiento $\left(5,10,20 \mathrm{y} 40^{\circ} \mathrm{C} / \mathrm{min}\right)$, calentando desde temperatura ambiente hasta $800^{\circ} \mathrm{C}$ en atmósfera inerte, con un flujo de nitrógeno constante de $100 \mathrm{~mL} / \mathrm{min}$, y una muestra entre 10 y $12 \mathrm{mg}$, que se alimentó al crisol de aluminio. A partir de los datos entregados por el equipo, empleando termogravimetría dinámica o termogravimetría no isotérmica, se obtuvieron las gráficas de peso remanente y velocidad de pérdida de peso en función de la temperatura, para cada velocidad de calentamiento a cada uno de los residuos. De las curvas de pérdida de peso (TG) y de velocidad de pérdida de peso (DTG), se obtuvieron las temperaturas características (temperatura inicial, máxima y final de degradación), la velocidad máxima de pérdida de peso y se determinó el índice de pirólisis. Este último parámetro es un índice de comparación entre diferentes materiales que expresa la reactividad o capacidad de la materia a ser pirolizada. De tal manera que un bajo valor de este índice indica que el material tiene una menor reactividad o capacidad de ser pirolizado, que aquel que muestra un mayor valor. Este índice depende de variables como tamaño de partículas y características físicas y químicas del residuo, que afectan la trasferencia de calor y masa, lo cual se establece indirectamente con las temperaturas características y la conversión. El índice de pirolisis se calcula por medio de la siguiente ecuación 3.

$$
I_{p}=\left(\frac{d \alpha}{d t}\right)_{\max } \cdot \frac{\alpha_{\Delta T}}{T_{m} \cdot T_{t} \cdot T_{f}}
$$

Donde $I_{p}$ es el índice de pirólisis $\left(\%{ }^{2} \cdot /\left(\mathrm{s} \cdot{ }^{\circ} \mathrm{C}^{3}\right),(\mathrm{d} \alpha / \mathrm{dt})_{\max }(\% / \mathrm{s})\right.$ la máxima velocidad de pérdida de peso, $T_{m}\left({ }^{\circ} \mathrm{C}\right)$ la temperatura a la máxima velocidad de pérdida de peso, $T_{i}$ la temperatura inicial de degradación y $T_{f}$ la temperatura final de degradación. Las temperaturas inicial y final de degradación se obtienen a conversiones de 5 y $95 \%$ respectivamente, $T_{m}$ se obtiene a $(\mathrm{d} \alpha / \mathrm{dt})_{\max }$ y $\alpha_{\Delta T}(\%)$ es la diferencia de conversiones al inicio y al finalde la etapa de degradación y tiene un 
valor de $90 \%$, el cual es fijado por Deng et al. [3].

De la información proporcionada por el análisis termogravimétrico se determinaron otros parámetros de pirólisis como el tiempo y la diferencia de temperatura para que el residuo se degrade desde un 5 a un $95 \%$. Estos parámetros indican qué residuo requiere un mayor tiempo de residencia en el proceso de pirólisis.

\subsection{Estudio cinético de los residuos}

El método isoconversional de Starink para la determinación del triplete cinético (energía de activación $(E)$, factor de frecuencia $(A)$, y orden de reacción $(n)$ ) de la degradación térmica de los residuos sólidos, es una aproximación desarrollada a partir de la combinación de los métodos de Flynn-Wall-Ozawa (FWO) [13] y Kissinger-Akahira-Sunose (KAS) [14], cuyas expresiones matemáticas se muestran en las ecuaciones 2 y 3 , respectivamente.

$$
\begin{gathered}
\operatorname{In} \beta_{i}=\left[\frac{A E}{R G(\alpha)}\right]-2,315-0,4567 \frac{E}{R T}=C_{0}-0,4567 \frac{E}{R T} \\
\operatorname{In}\left(\frac{\beta_{i}}{T_{p i}^{2}}\right)=\operatorname{In}\left(\frac{A_{k} R}{E_{k}}\right)-\frac{E_{k}}{R T_{p i}}=C_{k}-\frac{E_{k}}{R T_{p i}}
\end{gathered}
$$

El desarrollo de la ecuación de Starink se basa en el hecho que las expresiones de los métodos FWO y KAS, se pueden reescribir de una misma forma general, dada por la ecuación para la descomposición térmica en estado sólido, así [10] [12]:

$$
\operatorname{In}\left(\frac{\beta}{T^{1,8}}\right)=C_{S}-1,0037 \frac{E}{R T}
$$

Aquí $\beta$ es la velocidad de calentamiento, $R$ es la constante universal de los gases, $C_{S}$ es una constante y $T$ es la temperatura absoluta. Para una conversión dada la anterior ecuación se grafica, obteniendo una línea recta con pendiente $-1.0037 \mathrm{E} / \mathrm{R}$, de la cual se calcula el valor de $E$. Esta ecuación, al igual que la ecuación de los métodos FWO y KAS, considerando que la velocidad de degradación es función de un término que depende de la temperatura $(k(T))$, conocido como la constante de reacción, y un término que es función de la conversión, la cual esta elevada a una potencia conocida como orden de reacción [12].

El método Starink se trabaja con la conversión del residuo, $\alpha$, calculada mediante la ecuación 5, donde $W_{0}, W_{t}$ y $W_{\infty}$ son las masas de la muestra al inicio, al tiempo $t$ y al final de la reacción, respectivamente.

$$
\alpha=\frac{W_{0}-W_{t}}{W_{0}-W_{\infty}}
$$

Esta conversión se obtiene de pesos remanentes de la muestra registrados por el TGA. Con estos datos y la ecuación 4 se procede a determinar el triplete cinético, así: i) se calcula la energía de activación fijando diferentes valores de conversión (entre 0 y 1), ii) para cada valor fijo de conversión se lee la temperatura a cada una de las cuatro velocidades de calentamiento, iii) con estos datos se prepara un gráfico de $\ln \left(\beta / T^{1,8}\right)$ versus $1 / T$, obteniéndose una línea recta con pendiente 
$-1,0037 E / R T$, según la ecuación 4, de donde se calculada la energía de activación. Para determinar el orden de reacción y el factor de frecuencia, se utiliza la teoría de Avrami, también conocida como cinética de transformación de Johnson-Mehl-Avrami-Kolmogorov (JMAK), la cual describe el grado en que se transforma un material durante una cierta transformación de fase, como una función de la temperatura y el tiempo. El modelo JMAK originalmente se estableció para materiales cristalinos que cambian de fase mediante modelos cinéticos de nucleación y crecimiento, lo cual inicialmente limitaba su uso para la transformación de fase de materiales amorfos en general [15]. Sin embargo, la teoría de Avrami se ha implementado tanto para determinar órdenes de reacción en reacciones en estado sólido en sistemas isotérmicos y no isotérmicos de materiales amorfos y no amorfos, como en otras transformaciones de fase en estado sólido [16]. Una forma como se puede escribir la teoría de Avrami, considerando un modelo de orden de reacción, es por medio de la ecuación [15]:

$$
\alpha=1-\exp \frac{-k(T)}{\beta^{n}}
$$

Donde $k(T)$ es la constante de reacción dada por la ecuación de Arrhenius, la cual es:

$$
k(T)=A \exp \left(-\frac{E}{R T}\right)
$$

La constante de reacción es función de la temperatura y del factor de frecuencia o factor preexponencial A. Sustituyendo la ecuación 7 en la ecuación 6 y tomando logaritmos se obtiene:

$$
\operatorname{In}(-\operatorname{In}(1-\alpha))=\operatorname{In} A-\frac{E}{R T}-n \operatorname{In} \beta
$$

A continuación, se procede a: iv) fijar una temperatura $T$, v) a esta temperatura se lee la conversión para cada velocidad de calentamiento, vi) se realiza un gráfico de $\ln (-\ln (1-\alpha))$ versus $\ln \beta$ y se obtiene una línea recta con pendiente $-n$, de donde se determina el orden de reacción y del intercepto se calcula factor de frecuencia, según la ecuación 8 [15].

\section{Resultados y discusión de resultados}

\subsection{Caracterización fisicoquímica de los residuos}

Los resultados del análisis inmediato en base seca de los residuos veterinarios se presentan en la tabla I. En esta tabla se observa que la jeringa tiene el mayor contenido de materia volátil (MV), mayor poder calorífico (PCS y PCI) y menor contenido de carbono fijo (CF), cenizas (Cz) y humedad (Hw) en base húmeda, esto se debe a que el cuerpo de la jeringa está fabricado principalmente de Poliestireno. Mientras que el hisopo presenta el menor contenido de MV, el de mayor contenido de $\mathrm{CF}$ y el segundo en mayor contenido de $\mathrm{Cz}(0.80 \%)$. También se aprecia que todos los residuos presentan similares valores de PCS excepto la jeringa y la muestra denominada mezcla. El algodón se caracteriza por tener el mayor contenido de Hw. Estos resultados confirman cuán importante es trabajar en la estrategia conocida como "residuos a energía", actualmente implementada en el área de tratamiento de residuos en general, llevando a un máximo aprovechamiento del contenido energético de ellos [17]. 
Tabla I. Análisis inmediato de los residuos veterinarios en base seca (porcentaje en peso)

\begin{tabular}{ccccccc}
\hline Muestra & Hw & C & MV & CF & PCS(kJ/kg) & PCI(kJ/kg) \\
\hline Mezcla & 9.09 & 0.51 & 84.81 & 14.68 & 30077 & 27682 \\
\hline Jeringa & 0.15 & 0.23 & 99.77 & 0.00 & 45410 & 42354 \\
\hline Pelo & 13.21 & 0.54 & 78.80 & 20.66 & 21199 & 19273 \\
\hline Uñas & 11.39 & 0.99 & 78.65 & 20.36 & 21741 & 19890 \\
\hline Algodón & 23.91 & 0.54 & 87.11 & 12.35 & 21913 & 19699 \\
\hline Hisopo & 10.03 & 0.80 & 77.38 & 21.82 & 21446 & 19990 \\
\hline Gasa & 21.75 & 0.35 & 89.12 & 10.53 & 21774 & 19801 \\
\hline
\end{tabular}

En el análisis elemental, que se muestra en la tabla II, se aprecia que el residuo de jeringas muestra el mayor contenido de carbono $(\mathrm{C})$ e hidrógeno $(\mathrm{H})$ y los menores contenidos de azufre (S) y oxígeno $(\mathrm{O})$. Esto es debido al origen petroquímico del Poliestireno. Los residuos de jeringa y gasa no reportan contenido de nitrógeno $(\mathrm{N})$ en su composición. El pelo y las uñas muestran los mayores contenidos de $\mathrm{N}$, al igual que similares valores de los demás elementos, esto posiblemente se debe a que son residuos de origen animal. También se observa que el algodón, el hisopo y las gasas tienen similar análisis elemental, esto se debe a que los 3 están fabricados a base de fibra de algodón. Estos resultados complementan los análisis reportados por Deng et al. [3] y Zhu et al. [8], para residuos médicos en general.

Tabla II. Análisis elemental de los residuos veterinarios en base seca, libre de cenizas (porcentaje en peso)

\begin{tabular}{llllll}
\hline Muestra & $\mathbf{C}(\boldsymbol{\%})$ & $\mathbf{H}(\boldsymbol{\%})$ & $\mathbf{N}(\boldsymbol{\%})$ & $\mathbf{S}(\boldsymbol{\%})$ & $\mathbf{O ( \% )}$ \\
\hline Mezcla & 66.73 & 9.89 & 1.43 & 1.80 & 20.15 \\
\hline Jeringa & 84.35 & 13.88 & 0.00 & 0.13 & 1.63 \\
\hline Pelo & 48.26 & 6.98 & 16.24 & 5.53 & 22.99 \\
\hline Uñas & 50.03 & 7.04 & 16.70 & 3.70 & 22.54 \\
\hline Algodón & 45.34 & 6.58 & 1.42 & 0.12 & 46.54 \\
\hline Hisopo & 50.63 & 6.19 & 0.20 & 0.19 & 42.79 \\
\hline Gasa & 49.69 & 5.89 & 0.00 & 0.20 & 44.21 \\
\hline
\end{tabular}

\subsection{Perfiles de pirólisis de los residuos}

Las curvas TG de los residuos veterinarios se muestran en la figura 1, donde en el eje de las ordenadas se ubica el porcentaje de pérdida de peso o peso remanente (\%), todas normalizadas a un valor inicial de $100 \%$ para facilitar la comparación, y en el eje de las abscisas se grafica la temperatura en ${ }^{\circ} \mathrm{C}$. En esta figura se observa que las temperaturas inicial y final de descomposición de todos los residuos son diferentes. Lo cual se aprecia en el cambio de pendiente de las líneas horizontales al inicio y al final de cada curva TG, respectivamente. Esto se atribuye a la diferencia en las características de pirólisis de cada uno de los residuos. En esta figura también se observa que entre 200 y $350^{\circ} \mathrm{C}$ los residuos de pelo, uñas e hisopos sufrieron una importante pérdida de peso de aproximadamente el $70 \%$ respecto al peso inicial, mientras que los residuos de gasa y algodón experimentan su degradación entre 300 y $400^{\circ} \mathrm{C}$. Los residuos de jeringa muestran una pérdida de peso entre 410 y $470^{\circ} \mathrm{C}$. A $550^{\circ} \mathrm{C}$ todas las muestras completaron su descomposición por pirólisis. También se aprecia que la gasa y el algodón tienen curvas de pérdida de peso similares, esto debido la composición de la gasa en su mayoría es algodón. De igual manera se observa que el residuo 
de mayor pérdida de peso fue la jeringa, la cual experimentó la degradación térmica a mayores temperaturas comparado con los otros residuos.

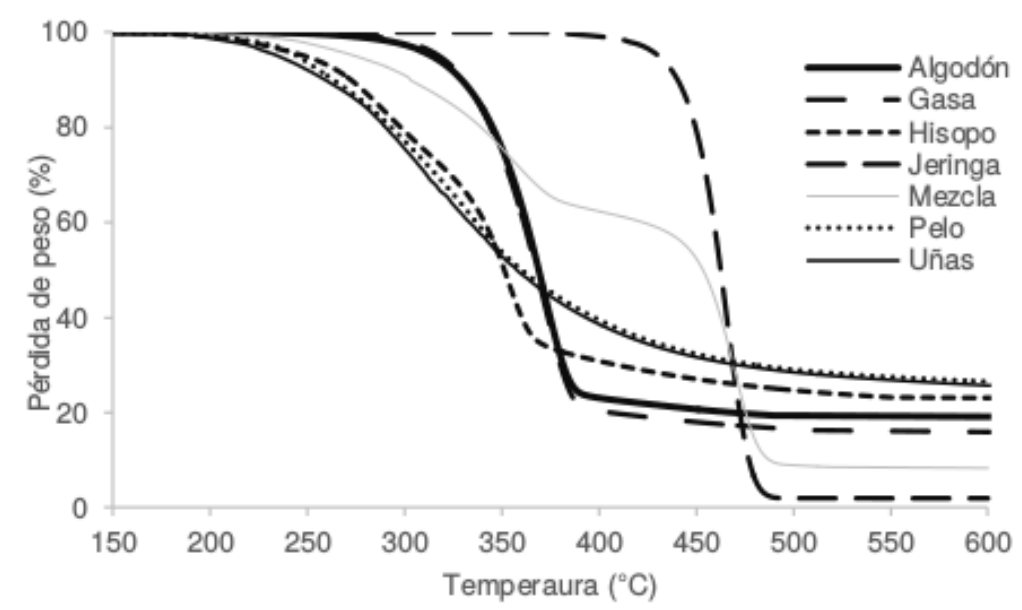

Figura 1. Curvas TG de los residuos veterinarios a $20^{\circ} \mathrm{C} / \mathrm{min}$.

Las curvas de la termogravimetría diferencial (DTG) de los residuos veterinarios se presentan en la figura 2. En esta figura se observa, entre $200 \mathrm{y} 300^{\circ} \mathrm{C}$, dos picos en las muestras de uñas, pelo y hisopo. Lo anterior puede indicar que existen dos etapas de degradación térmica, determinadas por la composición química y física individual de cada material analizado, lo cual juega un papel importante en su comportamiento en la pirólisis. Sin embargo, es importante resaltar que un mismo pico se puede deconvolucionar en varios picos que indicarían diferentes etapas en un mismo mecanismo de reacción. Los residuos de jeringa, gasa y algodón, solo muestran un pico, indicando que ellos se degradan bajo un único modelo de reacción. También se observa que las muestras de uñas y pelo, tienen comportamiento de degradación térmica similares, al igual que ocurre entre las muestras de hisopo, gasa y algodón. Esto se aprecia por las similitudes en el rango de temperatura de inicio y final de los picos, y que las curvas DTG de los residuos de gasa y algodón se encuentra superpuestas. De igual manera, se puede concluir que las muestras de uñas y pelo, se degradan con mayor facilidad, mientras que las muestras del cuerpo de la jeringa no se degradan tan fácilmente, debo a que requieren una mayor temperatura, comparado con los demás residuos. Esto concuerda con resultados reportados en la literatura para residuos médicos [3]. Respecto a la máxima velocidad de degradación, se observa que los residuos de jeringa, gasa, algodón e hisopo, experimentan en forma descendente, las mayores velocidades de degradación térmica en pirólisis de todos los residuos.

La figura 3 muestra la variación de las curvas DTG con la velocidad de calentamiento para el residuo algodón. Aquí se observa que la máxima velocidad de degradación del algodón aumenta disminuye al aumentar la velocidad de calentamiento. Además, la curva DTG se desplaza, alejándose del eje de las ordenadas con el aumento en la velocidad de calentamiento. A pesar que aquí no se muestra, este mismo comportamiento se observó para los otros residuos incluida la muestra denominada mezcla. Similares resultados fueron reportados por Slopiecka et al. [18] para la pirólisis de madera de álamo. 


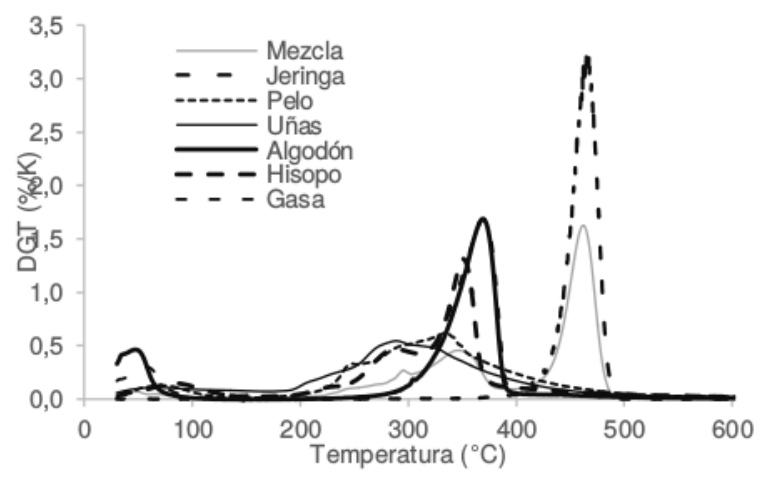

Figura 2. Curvas DTG de los residuos veterinarios a $20^{\circ} \mathrm{C} / \mathrm{min}$.

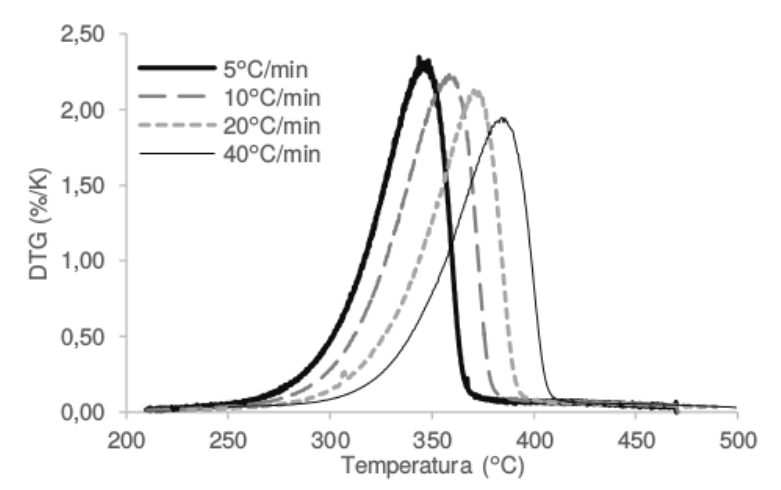

Figura 3. Variación de las curvas DTG de algodón a diferentes velocidades de calentamiento.

\subsection{Temperaturas características}

De las curvas obtenidas para cada residuo a las cuatro velocidades de calentamiento por la termogravimetría diferencial (DTG), se determinan las temperaturas características del perfil de pirólisis, las cuales se presentan en las figuras 4, 5 y 6, para las temperaturas $T_{i}, T_{m}$ y $T_{f}$ de degradación, respectivamente. En la figura 4 se observa que para la mayoría de los residuos la $T_{i}$ aumenta con la velocidad de calentamiento. También se puede ver que, en promedio para las cuatro velocidades de calentamiento, el residuo de jeringa presenta el mayor valor de $T_{i}\left(423^{\circ} \mathrm{C}\right)$, seguido por algodón $\left(303^{\circ} \mathrm{C}\right)$, gasa $\left(293^{\circ} \mathrm{C}\right)$, mezcla $\left(268^{\circ} \mathrm{C}\right)$, pelo $\left(237^{\circ} \mathrm{C}\right)$, hisopo $\left(234^{\circ} \mathrm{C}\right)$ y uñas $\left(217^{\circ} \mathrm{C}\right)$. De igual manera se observa que en términos generales al aumentar la velocidad de calentamiento se aprecia un incremento de las temperaturas características, que fácilmente se puede evidenciar con un desplazamiento hacia la derecha de las curvas que describen la descomposición de los residuos. Similares resultados son registrados por Zhu et al. [8], para papel adsorbente, algodón, aparatos plásticos de respiración y copitos de madera.

Para la temperatura máxima de pérdida de peso, que se muestra en la figura 5, se aprecia que el orden de los residuos de mayor a menor $T_{m}$ es: jeringa $\left(461^{\circ} \mathrm{C}\right)$, mezcla $\left(435^{\circ} \mathrm{C}\right)$, algodón $\left(365^{\circ} \mathrm{C}\right)$,

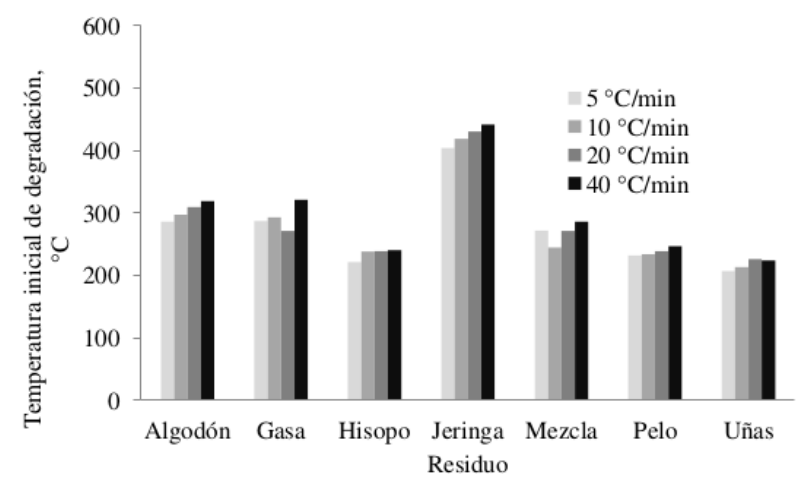

Figura 4. Variación de la temperatura inicial de degradación para residuos veterinario a diferente velocidad de calentamiento.

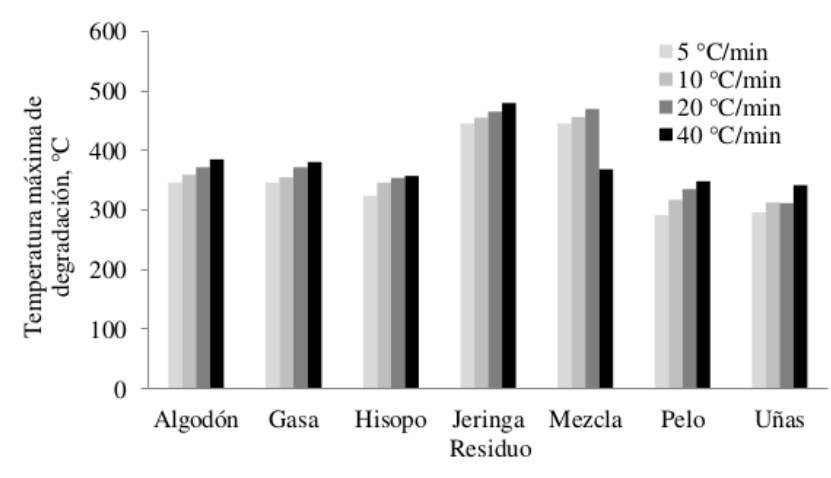

Figura 5. Variación de la temperatura máxima de degradación para los residuos veterinarios a diferente velocidad de calentamiento. 


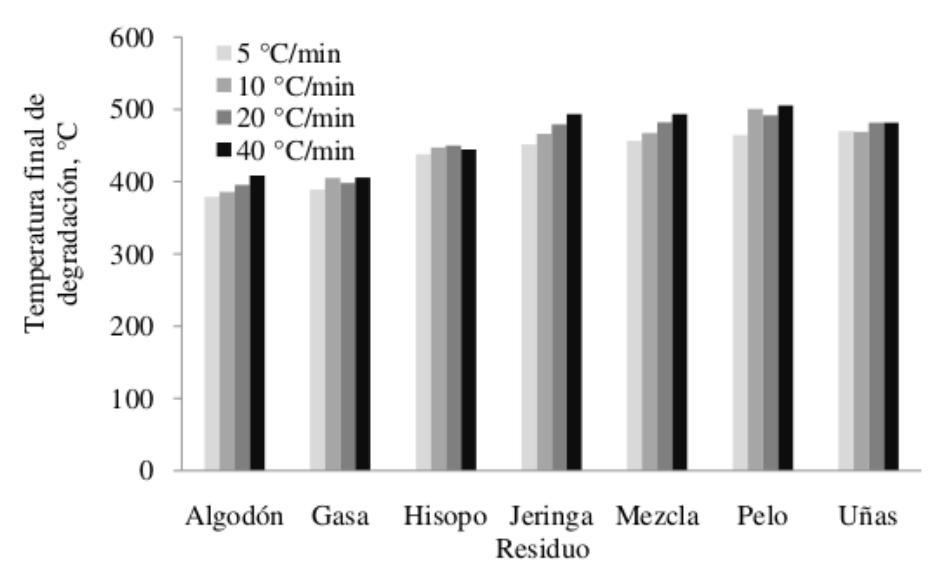

Figura 6. Variación de la temperatura final de degradación para los residuos veterinarios a diferente velocidad de calentamiento.

gasa $\left(363^{\circ} \mathrm{C}\right)$, hisopo $\left(345^{\circ} \mathrm{C}\right)$, pelo $\left(323^{\circ} \mathrm{C}\right)$ y uñas $\left(315^{\circ} \mathrm{C}\right)$. Respecto a la temperatura final de degradación (figura 6 ) se observa que, en promedio para las cuatro velocidades de calentamiento, la $T_{f}$ mayor es para el residuo de pelo $\left(490^{\circ} \mathrm{C}\right)$, valores similares para los residuos de jeringa $\left(472^{\circ} \mathrm{C}\right)$, mezcla $\left(474^{\circ} \mathrm{C}\right)$ y uñas $\left(475^{\circ} \mathrm{C}\right)$, seguido por hisopo $\left(444^{\circ} \mathrm{C}\right)$, gasa $\left(399^{\circ} \mathrm{C}\right)$ y algodón $\left(391^{\circ} \mathrm{C}\right)$. Si se toma las temperaturas $T_{i}$, y $T_{m}$ como parámetros cualitativos para establecer la reactividad a la pirólisis de los residuos veterinarios, considerando el más reactivo aquel que presente el menor valor de estos parámetros, se puede decir que los tres residuos de mayor reactividad son: uñas, hisopo y pelo, y los dos de menos reactivos son: jeringa y algodón.

\section{4. Índice de pirólisis}

Índice de Pirólisis a diferentes velocidades de calentamiento para los 7 residuos se presentan en la tabla III.

Tabla III. Índice de Pirólisis a diferentes velocidades de calentamiento $\left(10^{-6}\right)$

\begin{tabular}{cccccc}
\hline Muestra & $\mathbf{5}^{\circ} \mathbf{C} / \mathbf{m i n}$ & $\mathbf{1 0}^{\circ} \mathbf{C} / \mathbf{m i n}$ & $\mathbf{2 0}^{\circ} \mathbf{C} / \mathbf{m i n}$ & $\mathbf{4 0}^{\circ} \mathbf{C} / \mathbf{m i n}$ & $\mathbf{r}^{2}$ \\
\hline Mezcla & 2.50 & 3.35 & 10.48 & 13.36 & 0.886 \\
\hline Jeringa & 4.74 & 8.43 & 14.95 & 26.47 & 0.998 \\
\hline Pelo & 1.70 & 2.65 & 5.27 & 11.18 & 0.996 \\
\hline Uñas & 1.89 & 5.57 & 6.95 & 10.84 & 0.916 \\
\hline Algodón & 4.21 & 7.48 & 14.66 & 24.91 & 0.993 \\
\hline Hisopo & 3.53 & 5.86 & 11.90 & 23.37 & 0.999 \\
\hline Gasa & 4.36 & 7.35 & 14.80 & 24.76 & 0.992 \\
\hline
\end{tabular}

La última columna de la tabla es el coeficiente de correlación lineal, el cual muestra la relación existente entre el $I_{p}$ y la velocidad de calentamiento. En todos los residuos se observa que el $I_{p}$ aumenta con la velocidad de calentamiento en forma lineal, excepto para la mezcla que presenta un $r^{2}$ de 0.886 . Al comparar el $I_{p}$ a cualquier velocidad de calentamiento, los residuos con mayor capacidad a ser pirolizados son jeringa, algodón y gasa, seguidos por hisopo, mezcla, uñas y pelo. Lo anterior indica que no necesariamente los residuos que son más reactivos a la pirólisis (que inicien la pirólisis a baja temperatura), sean los que tengan la mayor capacidad a ser pirolizados. 
Estos resultados son comparables con los presentados en la literatura para residuos médicos [3].

\subsection{Otros parámetros de degradación}

En la tabla IV se muestra los valores de otros parámetros característicos del proceso de pirólisis, obtenidos por medio del análisis termogravimétrico a diferentes velocidades de calentamiento. Estos parámetros son: $(d \alpha / d T)_{m},(d \alpha / d T)_{1 / 2}, \Delta T, \alpha_{m}$, los cuales representan la velocidad máxima de conversión o de pérdida de peso o velocidad máxima de degradación debido a la reacción de pirólisis, la velocidad de conversión a la mitad de la altura del pico, la diferencia de temperatura para que un material se degrade desde un 5 hasta un $95 \%$, y la conversión para la máxima velocidad de reacción de degradación, respectivamente.

Tabla IV. Parámetros característicos de pirólisis: velocidad máxima de degradación y rango de temperatura de degradación

\begin{tabular}{|c|c|c|c|c|c|c|c|c|c|c|c|c|c|c|c|c|}
\hline \multirow[b]{2}{*}{ Residuo } & \multicolumn{4}{|c|}{$5^{\circ} \mathrm{C} / \mathrm{min}$} & \multicolumn{4}{|c|}{$10^{\circ} \mathrm{C} / \mathrm{min}$} & \multicolumn{4}{|c|}{$20^{\circ} \mathrm{C} / \mathrm{min}$} & \multicolumn{4}{|c|}{$40^{\circ} \mathrm{C} / \mathrm{min}$} \\
\hline & $\begin{array}{l}(d \alpha / d T)_{m} \\
\% / \mathrm{K}\end{array}$ & $\begin{array}{l}(d \alpha / d T)_{1 / 2} \\
\% / \mathrm{K}\end{array}$ & $\Delta T,{ }^{\circ} \mathrm{C}$ & $\begin{array}{l}\alpha_{m} \\
\%\end{array}$ & $\begin{array}{l}(d \alpha / d T)_{m} \\
\% / \mathrm{K}\end{array}$ & $\begin{array}{l}(d \alpha / d T)_{1 / 2} \\
\% / \mathrm{K}\end{array}$ & $\Delta T,{ }^{\circ} \mathrm{C}$ & $\begin{array}{l}\alpha_{m} \\
\%\end{array}$ & $\begin{array}{l}(d \alpha / d T)_{m} \\
\% / \mathrm{K}\end{array}$ & $\begin{array}{l}(d \alpha / d T)_{1 / 2} \\
\% / \mathrm{K}\end{array}$ & $\Delta T,{ }^{\circ} \mathrm{C}$ & $\begin{array}{l}\alpha_{m} \\
\%\end{array}$ & $\begin{array}{l}(d \alpha / d T)_{m} \\
\% / \mathrm{K}\end{array}$ & $\begin{array}{l}(d \alpha / d T)_{1 / 2} \\
\% / \mathrm{K}\end{array}$ & $\Delta T,{ }^{\circ} \mathrm{C}$ & $\begin{array}{l}\alpha_{m} \\
\%\end{array}$ \\
\hline Jeringa & 4.13 & 2.06 & 47.63 & 76.98 & 3.76 & 1.88 & 46.99 & 68.99 & 3.34 & 1.66 & 48.77 & 59.40 & 3.16 & 1.59 & 52.08 & 61.35 \\
\hline Algodón & 2.31 & 1.16 & 92.83 & 64.94 & 2.24 & 1.11 & 88.10 & 67.67 & 2.17 & 1.09 & 86.15 & 67.79 & 1.95 & 0.98 & 88.90 & 68.40 \\
\hline Gasa & 2.71 & 1.36 & 101.44 & 66.80 & 2.09 & 1.04 & 111.91 & 54.54 & 2.01 & 1.01 & 126.71 & 67.78 & 1.94 & 0.97 & 84.37 & 64.72 \\
\hline Mezcla & 1.82 & 0.91 & 184.40 & 80.36 & 1.73 & 0.87 & 222.06 & 85.77 & 1.56 & 0.78 & 210.22 & 77.36 & 1.07 & 1.04 & 207.37 & 74.65 \\
\hline Hisopo & 1.95 & 0.98 & 216.64 & 66.47 & 1.58 & 0.79 & 208.10 & 68.62 & 1.53 & 0.77 & 211.44 & 68.33 & 1.50 & 1.25 & 203.43 & 68.63 \\
\hline Pelo & 0.86 & 0.43 & 232.12 & 33.45 & 0.85 & 0.43 & 266.58 & 46.48 & 0.78 & 0.39 & 253.11 & 52.75 & 0.71 & 0.36 & 258.27 & 53.26 \\
\hline Uñas & 0.89 & 0.45 & 263.11 & 42.94 & 0.80 & 0.39 & 255.97 & 44.05 & 0.72 & 0.37 & 255.00 & 40.92 & 0.70 & 0.35 & 257.40 & 43.13 \\
\hline
\end{tabular}

En esta tabla se observa que, para cualquier velocidad de calentamiento, la diferencia de temperatura para que un residuo se descomponga desde un 5 a un $95 \%$, varía según el tipo de residuo. Por lo tanto, el orden de menor a mayor diferencia de temperatura requerida para que ocurra la degradación de los residuos veterinarios es: jeringa, algodón, gasa, mezcla, hisopo, pelo y uñas. Este mismo orden de los residuos se cumple para valores de velocidad máxima de degradación de mayor a menor. Estos resultados muestran que los residuos a base de fibra de algodón están seguidos unos de otros, al igual que ocurre con los residuos de origen animal (pelo y uñas). También se aprecia que la velocidad máxima de degradación y velocidad de conversión a la mitad de la altura del pico, disminuyen con el incremento de la velocidad de calentamiento. Mientras que la conversión para la máxima velocidad de reacción de degradación no tiene una relación directa con el aumento de la velocidad de calentamiento, dado que para algunos esta conversión aumenta (algodón, hisopo, pelo), para otros disminuye (jeringa, mezcla) y en otros no hay una tendencia clara (gasa, pelo).

\subsection{Análisis cinético de los residuos}

De la pendiente de la ecuación 1 se obtiene la energía de activación a una conversión fija. La variación de $E$ con la conversión se muestra en la figura 7. En esta figura se observa que $E$ no cambia significativamente con la conversión para los residuos de jeringa, gasa y algodón, indicando que se degradan bajo un único mecanismo de reacción. Sin embargo, la $E$ varía poco para el hisopo, y se presentan los mayores cambios de E con la conversión para uñas y pelo. A conversiones superiores al $50 \%$ la variación de $E$ es más notoria, generándose un cambio de pendiente alrededor del $50 \%$ para el pelo y de $60 \%$ para las uñas. Este cambio de pendiente confirma que dichos residuos se degradan por medio de un mecanismo complejo de múltiples etapas y que el mecanismo de reacción no es el mismo en todo el proceso de descomposición [19], como se analizó en la figura 2. Sin embargo, se debe tener cuidado cuando se presentan más de dos picos en un diagrama DTG, 
dado que un mismo pico se puede deconvolucionar en varios picos que indicarían diferentes etapas en un mismo mecanismo de reacción.

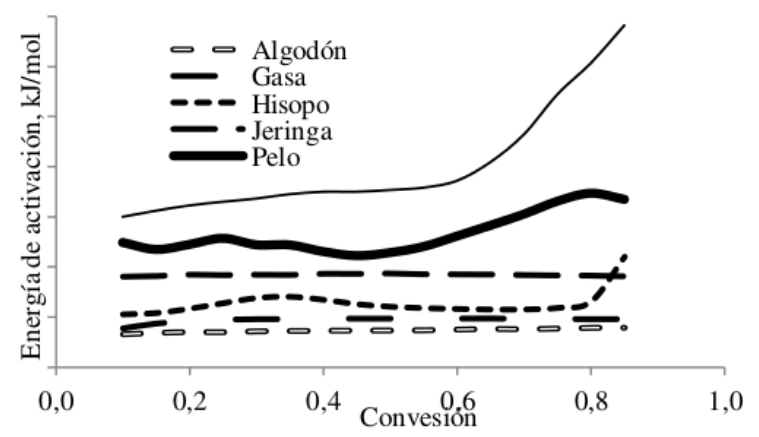

Tabla V. Triplete cinético de los residuos veterinarios por el método de Starink

\begin{tabular}{ccccc}
\hline Muestra & $\mathbf{E}(\mathbf{k J} / \mathbf{m o l})$ & $\mathbf{n}$ & $\mathbf{I n} \mathbf{A}$ & $\mathbf{r}^{2}$ \\
\hline Algodón & 172.96 & 0.78 & 31.81 & 0.9995 \\
\hline Gasa & 173.09 & 0.86 & 31.75 & 0.9896 \\
\hline Hisopo & 189.57 & 0.36 & 37.29 & 0.8347 \\
\hline Jeringa & 223.26 & 1.27 & 34.53 & 0.9993 \\
\hline Pelo & 251.01 & 0.23 & 47.54 & 0.9593 \\
\hline Uñas & 271.12 & 0.25 & 53.43 & 0.9268 \\
\hline
\end{tabular}

Figura 7. Variación de la energía de activación con la conversión.

La energía de activación en cada residuo se promedió para el rango de conversiones entre 1 y $99 \%$, el factor frecuencia (Ln A) y el orden de reacción se calcularon de la pendiente y el intercepto de la ecuación (8), respectivamente. Estos resultados al igual que el coeficiente de correlación se presentan en la tabla V. La energía de activación es: $E_{\text {uñas }}>E_{\text {pelo }}>E_{\text {jeringa }}>E_{\text {hisopo }}>E_{\text {gasa }}>$ $E_{\text {algodón, }}$, esto indica que los residuos de algodón son más reactivos a la pirólisis que los residuos de uñas. El orden de reacción es: $n_{\text {pelo }}>n_{\text {uñas }}>n_{\text {hisopo }}>n_{\text {algodón }}>n_{\text {gasa }}>n_{\text {jeringa }}$. En esta tabla también se observa que los coeficientes de correlación son cercanos a la unidad, excepto para el hisopo.

\section{Conclusiones}

El poder calorífico para los residuos de gasa, algodón, pelo, uñas e hisopo, presenta valores muy cercanos entre ellos. Para la jeringa se encontró un valor de poder calorífico dos veces mayor en comparación con los residuos anteriores, lo cual se debe a que se encuentra hecha en su mayor parte de Poliestireno. Es por ello que para la jeringa se requiere alcanzar una mayor temperatura para poder lograr su pirólisis. Caso contrario con los residuos de algodón, gasa, hisopo, uñas y pelo pirolizan a temperaturas menores de $400^{\circ} \mathrm{C}$.

El método de Starink presenta una buena correlación de las variables de seguimiento en el estudio de la pirólisis de los residuos veterinarios de algodón, jeringa, gasa, pelo, uñas y en menor medida para el hisopo. Encontrándose que, por medio de este método, los residuos de algodón son los más reactivos a la pirólisis, seguido de los residuos de gasa, hisopo, jeringa, pelo y uñas. Lo cual se establece con el orden de menor a mayor valor de la energía de activación $\left(E_{\text {algodón }}<E_{\text {gasa }}<E_{\text {hisopo }}<E_{\text {jeringa }}<E_{\text {pelo }}<E_{\text {uñas }}\right)$.

El orden de reacción de los residuos veterinarios se encuentra entre 0.23 y 1.27, la energía de activación están en el rango de 172 y $272 \mathrm{~kJ} / \mathrm{mol}$ y la probabilidad de colisión o factor exponencial entre 31 y 54. La validación de estos datos al igual que del modelo cinético, con miras a un modelado y escalado, será objeto de un estudio posterior. 
A partir del análisis termogravimétrico se estableció que los residuos veterinarios de uñas, pelo e hisopo, se degradan mediante dos diferentes mecanismos de reacción complejos de múltiples etapas, mientras los residuos de jeringa, gasa y algodón lo realizan bajo un único mecanismo, lo cual se confirma con el cambio de pendiente que se observa en las curvas de energía de activación versus conversión.

Los valores del índice de pirólisis y de poder calorífica sugieren que los residuos veterinarios, incluidos todos los residuos hospitalarios, podrían ser aprovechados en la generación de energía mediante combustión y pirólisis, proporcionando un desarrollo energético sostenible a ciudades en continuo crecimiento, que buscan un avance rural sustentable desde el punto de vista energético y ambiental.

\section{Agradecimientos}

Los autores expresan su más sinceros agradecimientos a la Universidad Nacional de Colombia por el apoyo económico al proyecto "Características de pirólisis y cinética de degradación de los residuos sólidos veterinarios" con código HERMES 17890, al Laboratorio de Combustión Combustibles de la Universidad del Valle por su apoyo a la realización de los análisis, y a las estudiantes de programa de Ingeniería Química Linna Gisseth Lucero Moran, Diana Marcela Hernández Gutiérrez y Estefany Ortiz Cardona, por el apoyo a la parte experimental del proyecto.

\section{Referencias}

[1] J.A Blanco y F.G. Briceño, Diseño de una planta de tratamiento de residuos sólidos hospitalarios y similares en el municipio de Arauca, capital. Trabajo de grado en Ingeniería Ambiental. Universidad Nacional de Colombia, sede Arauca, 2005.

[2] E.S. Windfeld y M.S. Brooks, "Medical waste management: A review". Journal of Environmental Management, Volumen 163, 2015, pp. 98-108.

[3] N. Deng,Y. Zhang y Y. Wang, "Thermogravimetric analysis and kinetic study on pyrolysis of representative medical waste composition”. Waste Management, Volumen 28, 2008, pp. 1572-1580.

[4] J.H. Yan, H.M. Zhu, X.G. Jiang, Y. Chi, y K.F. Cen, "Analysis of volatile species kinetics during typical medical waste materials pyrolysis using a distributed activation energy model", Journal of Hazardous Materials, Volumen 162, 2009, pp. 646-651.

[5] J.W. Bujak, "Thermal treatment of medical waste in a rotary kiln". Journal of Environmental Management, Volumen 162, 2015, pp. 139-147.

[6] C.C. Lee, y G.L. Huffman, "Medical waste management/incineration". Journal of Hazardous Materials, Volumen 48, 1996, pp. 1-30.

[7] Q. Wang, W. Zaho, H. Liu, C. Jia y H. Xu, "Reactivity and kinetic analysis of biomass during combustion". Energy Procedia, Volumen 17, 2012, pp. 169-875.

[8] H.M. Zhu, J.H. Yan, X.G. Jiang, Y.E. Lai y K.F. Cen, "Study on pyrolysis of typical medical waste materials by using TG-FTIR analysis". Journal of Hazardous Materials, Volumen 153, 2008, pp. 670-676.

[9] J.W. Bujak, "Heat recovery from thermal treatment of medical waste". Energy, Volumen 90, 2015, pp. 1721-1732.

[10] C. Gai, Y. Dong y T. Zhang, "The kinetic analysis of the pyrolysis of agricultural residue under non-isothermal conditions". Bioresource Technology, Volumen 127, 2013, pp. 298-305.

[11] A.P Gallego y J.E. Castro, "Sobre el rol innovador de la educación energética para la investigación en ingeniería". Ingeniería, Volumen 19 (2), 2014, pp. 147-163.

[12] S. Vyazovkin, A.K. Burnham, J.M. Criado, L.A. Perez-Maqueda, C. Popescu y N. Sbirrazzuoli, "ICTAC kinetics committee recommendations for performing kinetic computations on thermal analysis data". Thermochimica Acta, Volumen 520, 2011, pp. 1-19. 
[13] Q. Xu, X. Ma, Z. Yu y Z. Cai, "A kinetic study on the effects of alkaline earth and alkali metal compounds for catalytic pyrolys is of microalgae using thermogravimetry". Applied Thermal Engineering, Volumen 73, 2014, pp. 355-359.X.

[14] X. Peng, X. Ma y Z. Xu, "Thermogravimetric analysis of co-combustion between microalgae and textile dyeing sludge". Bioresource Technology, Volumen 180, 2015, pp. 288-295.

[15] G. Ruitenberg, E. Woldt y A.K. Petford-Long, "Comparison the Johnson-Mehl-Avrami-Kolmogorov equations for isothermal and linear heating conditions". Thermochimica Acta, Volumen 378, 2001, pp. 97-105.

[16] M. Tomellini, "Functional form of the Kolmogorov-Johnson-Mehl-Avrami kinetics for non-isothermal phase transformations at constant heating rate". Thermochimica Acta, Volumen 566, 2013, pp. 249-256.

[17] A. Tabasova, J. Kropac, V. Kermes, A. Nemet y P. Stehlík, "Waste-to-energy technologies: impact on environment". Energy, Volumen 44, 2012, pp.146-155.

[18] K. Slopiecka, P. Bartocciy F. Fantozzi, "Thermogravimetric analysis and kinetic study of poplar wood pyrolysis". Applied Energy, Volumen 97, 2012, pp.491-497.

[19] K. Slopiecka, P. Bartocci y F. Fantozzi. Thermal analysis and kinetic study of poplar Wood pyrolysis, Third International Conference on Applied Energy, Perugia, Italia, Mayo de 2011, pp. 1687-1698.

\section{Andrés Felipe Rojas González}

Ingeniero Químico, Universidad Nacional de Colombia, Manizales; magíster en Ingeniería Química, Universidad del Valle; doctor en Ingeniería, énfasis Ingeniería Química, Universidad del Valle; actualmente se desempeña como Profesor Asociado en Dedicación Exclusiva en el área de térmicas en la Universidad Nacional de Colombia, Manizales, Colombia, y hace parte del Grupo de Investigación en Aprovechamiento de Residuos de la Universidad Nacional sede Manizales y del Grupo de Investigación en Prospectiva Ambiental de la Universidad Nacional de la Sede Palmira; director del Grupo de Investigación en Aprovechamiento de Residuos - GIAR - donde realiza estudios sobre Valorización de Residuos y Director del Laboratorio de Aprovechamiento de Residuos.

Correo electrónico: anfrojasgo@unal.edu.co

\section{Laura María Aranzazu Ríos}

Ingeniera Química, Universidad Nacional de Colombia, Manizales, Colombia; actualmente se desempeña como Jefe de Control de Calidad en la Planta de Alcoholes Sulfatados en la empresa Químicos del Cauca, Cauca, Colombia; es integrante activa del Grupo de Aprovechamiento de Residuos, GIAR, de la Universidad Nacional de Colombia, Manizales, Colombia.

Correo electrónico: laurisma21@gmail.com 